\title{
Special Issue: Fuzzy logic systems for transportation engineering
}

\author{
Dalin Zhang ${ }^{\mathrm{a}}$, Sabah Mohammed ${ }^{\mathrm{b}}$ and Alessandro Calvi ${ }^{\mathrm{c}}$ \\ ${ }^{a}$ School of Software Engineering, Beijing Jiaotong University, Beijing, China \\ ${ }^{\mathrm{b}}$ Department of Computer Science, Lakehead University, Thunder Bay, Canada \\ ${ }^{\mathrm{c}}$ Università Roma Tre, Rome, Italy
}

Fuzzy logic is a figure of multi-valued logic, which derived from fuzzy set theory to deal with approximate reasoning rather than precise. A fuzzy logic approach application can optimize multiplex goals and then reach a nearer similarity to the real world. Modeling of incorrect scheduling knowledge is permitted by fuzzy logic, with linguistic variables, which defined via membership functions showing the accuracy degree of the data and the reasoning about the incorrect data by using fuzzy rules. Fuzzy logic that was introduced by Zadeh (1965) has been utilized to several industrial problems. Fuzzy logic system approach privilege is incorporating both numerical results from a previous solution or simulation and the scheduling expertise from experiences or observation, and that's convenient to implement.

Fuzzy logic is shown to be a very promising mathematical approach to modeling traffic and transportation processes characterized by subjectivity, ambiguity, uncertainty and imprecision. The basic premises of fuzzy logic systems are presented as well as a detailed analysis of fuzzy logic systems developed to solve various traffic and transportation engineering problems. Emphasis is put on the importance of fuzzy logic systems as universal approximators in solving traffic and transportation problems. Possibilities are shown

\footnotetext{
${ }^{*}$ Corresponding author. Dalin Zhang, School of Software Engineering, Beijing Jiaotong University, Beijing, China. E-mail: dalin@bjtu.edu.cn.
}

regarding the further application of fuzzy logic in this field.

This special section aims to provide a forum for researchers who address this issue and to present their work in a peer-reviewed forum. Authors are solicited to contribute to the conference by submitting articles that illustrate research results, projects, surveying works and industrial experiences that describe significant advances in the following areas, but are not limited to these topics only. The success of this Special Section is attributed to the timely research issue and the unfailing efforts of the Guest Editor. In general, the articles were mainly distributed into twenty-five categories.

- Fuzzy System Applications in Expressway

- Fuzzy System Applications in Urban Transportation

- Fuzzy System Applications in Rural Transportation

- Fuzzy System Applications in Rail Transportation

- Fuzzy System Applications in Air Transportation

- Fuzzy System Applications in Water Transportation

- Fuzzy System Applications in Logistics

- Fuzzy System Applications in Transportation Integration

- Fuzzy Decision Support System 
- Fuzzy Expert System

- Fuzzy Mathematical Programming

- Fuzzy Decision Making and Decision Support Systems

- Fuzzy Neural Systems, Neuro-fuzzy Systems

- Fuzzy Systems Modeling and Identification

- Fuzzy Pattern Recognition

- Fuzzy Process Control

- Fuzzy Reasoning System

- Fuzzy-rule Based System

- Fuzzy System Applications in Computer Vision

- Hybrid Fuzzy Systems (Fuzzy-neuroevolutionary-rough)

- Fuzzy Sets in Bioinformatics

- Fuzzy System Applications in Human-Machine Interface

- Fuzzy System Applications in Robotics

- Fuzzy System Applications in System and Control Engineering

Hence, we attempt to summarize the contributions of the 49 articles.

In the article "Network effect in shared supply chain platform value co-creation behavior in evolutionary game" by Zou et al., on the basis of a platform network effect perspective, this study constructed an evolutionary game model of value co-creation behavior for a shared supply chain platform and manufacturers, analyzed their evolutionary stable strategies, and used numerical simulation analysis to further verify the model. In addition, the boundary condition for the shared supply chain platform to actively participate in value co-creation was that the cost of the shared supply chain platform for active participation in value co-creation must be less than that of passive participation.

In the article "Evaluating operation efficiency of public transportation: a three-stage DEA method" by Jing Li et al., based on the traffic data of Beijing, this paper used the principal component analysis method to establish a reliable indicator system of efficiency evaluation. It then used the three-stage DEA model to evaluate the efficiency of rail transit and ground bus in 2008-2017, which described the development trend and effective situation of rail transit and ground bus in Beijing in the past ten years. Besides, it also compared and analyzed the calculated efficiency before and after the adjustment.

In the article "Layout evaluation for electric vehicle charging pile based on charging frequency" by Xiangke Cui et al., this study aimed to solve the problem of locating charging stations for public elec- tric vehicles. A Non-deterministic Polynomial model aiming to minimize the total vehicle service distance is developed. The authors used an agent-based model to simulate the optimized charging station location based on Anylogic. Through a case study of Beijing, they tested the model in five situations. The improved model has a shorter response time to passenger demand, shorter service time for passengers but more mileage for electric vehicles.

In the article "Bayesian assessment of utility tunnel risk based on information sharing mechanism" by Zhang et al., the authors used the Stackelberg game model to analyze the risk information sharing among utility tunnel institutions which concludes in utility tunnel company and pipeline company. On this basis, this paper analyzed the potential disaster risk factors in the operation process of utility tunnel, and constructed the risk early warning model of integrated pipeline corridor based on Bayesian network.

In the article "User logistics profiling for terminal distribution based on adaptive large adjacent search" by Li et al., the authors studied the problem of terminal delivery route planning user logistics profiles. It mainly generated user profiles from two aspects: consumers' preference for self-pickup services and consumers' complaint tendencies. Based on the results of user profiles, an Adaptive Large Adjacent Search algorithm was established to design the delivery route of terminal distribution and determine the appropriate delivery strategy to reduce delivery costs and improve customer satisfaction.

In the article "Subway passenger flow analysis and management optimization model based on AFC data" by Sun et al., the authors, based on the AFC data of Beijing subway system, analyzed the temporal and spatial distribution characteristics of the passenger flow in the subway network. At the same time, taking subway line 5 as an example, it quantitatively calculated the imbalance coefficient of passenger flow in time, section and direction. Combined with the calculation results, it also proposed management optimization model and gave some advice to the subway operation department from the aspects of passenger organization and transport management.

In the article "Applying an entropy method to evaluate regional traffic development from a niche perspective" by Zhang et al., the authors used a niche model and entropy method to analysis the development status of regional transportation industry. The study data were collected from the National Bureau of Statistics, and the "Statistical Bulletin of National Economy and Social Development". The niche model 
The model includes two aspects: "status" and "potential". The entropy method was used to determine the weight of each index.

In the article "A price discrimination based Cournot game model for high-speed rail and airlines" by Li et al., the authors established a multi-level price competition pricing Cournot model for high-speed rail and airline to shed light on the HSR-air transport competition impact of pricing discrimination when airlines could offer multi-level class seats, and analyzed the optimal pricing strategy of airlines and high-speed rail operators and their impact on social welfare. The analytical results demonstrated that airline price discrimination will increase social welfare and reduce consumer surplus, both airfare and airline profit increase as the degree of price discrimination increases welfare.

In the article "A decision-making model for railway tourist dedicated train operation plan" by Liang et al., the authors addressed the decisionmaking between the railway enterprises and the local government on whether the tourist-dedicated train stops at the local station, the length of stay and the market price, the model of its operation plan was designed to solve algorithm problem and provided foundation for railway enterprises to improve the efficiency of operation. Based on the results of experiments, the authors found that the price sensitivity of consumers is negatively correlated with the pricing and income of tourist-dedicated trains. In addition, a larger demand scale will increase the possibility of the tourist-dedicated train stopping.

In the article "The correlations of transportation with regional economy and demographic space" by Wu Xiaoping et al., the authors constructed a comprehensive evaluation model of transportation superiority, then select Southwest China as a case to evaluate the transportation superiority and analyze the correlations of transportation with regional economic development and population distribution. The results show that there is a positive correlation between regional transportation superiority and regional population density as well as GDP per capita in the spatial distribution. Finally, by using the fuzzy logic analysis method, we propose countermeasures and suggestions for the impact of transportation integration on regional economic development and population distribution.

In the article "LIRP optimization of cold chain logistics in "satellite warehouse" mode of supermarket chains" by Bo Shu et al., the authors proposed the model of Location Inventory Routing Problem (LIRP) optimization in "satellite warehouse" mode in view of customer satisfaction with the broken line soft time windows. The model minimizes the total cost of the cold chain logistics system of supermarket chain through the location allocation, inventory optimization, the determination of distribution service relationship between "satellite warehouse" and customer, and the constraint of time penalty cost. Then, the paper designed an improved ant colony optimization to solve the LIRP model of supermarket chain. Finally, the simulation in MATLAB verifies and analyzes the validity of the model and algorithm. Therefore, LIRP optimization model in "satellite warehouse" mode can effectively improve the operational efficiency of fresh products home-delivery in the supermarket chain and thus reduce the logistics cost.

In the article "Transportation Routes Evaluation Between China and Vietnam: A Delphi and CFPR Approach" by Lixin Shen et al., the authors scientifically evaluated the competing routes for the multimodal container transport supporting ChinaVietnam trade, analyzed eight transport routes from Chongqing to Hai Phong, and evaluated these routes with the Delphi and CFPR methods. This study can inform Chinese and Vietnamese trade enterprises in terms of selecting their preferred multimodal transport route strategies and the governmental authorities of both countries in making management decisions.

In the article "A Kalman filtering fuzzy logic algorithm for recognition of lane departure" by Kai Ren $e t$ $a l$. , the authors established a lane departure decisionmaking method without calibration relying on the Kalman filtering fuzzy logic algorithm, according to the characteristics of expressway lanes, based on the machine vision and hearing fusion analysis of lane departure, integrating the extraction of the linear lane line model and the region of interest (ROI) in the article to judge the degree of vehicle departure from the lane by integrating the slope values of the 2 lane lines in the road image. The results show that the system has good lane recognition capabilities and accurate departure decision-making capabilities, and meet the lane departure warning requirements in the expressway environment.

In the article "Decision-making support for transportation and logistics combining rough set fuzzy logic algorithm" by Tian Chen et al., the authors combined the rough set fuzzy logic algorithm with data mining to support the decision making based on the reverse logistics. Finally, they established a 
vehicle scheduling optimization model to support the decision making in transportation logistics.

In the article "A PMSM fuzzy logic regenerative braking control strategy for electric vehicles" by Feng Liu et al., the authors proposed a series regenerative braking control strategy based on PMSM fuzzy logic. According to this strategy, the motor braking shall be used as much as possible based on ensuring braking stability, 4 braking zones shall be divided according to the braking intensity, and different braking force distribution strategies shall be used, while comprehensively considering influencing factors such as vehicle speed, ECE regulations, battery, and motor characteristics. Simulink and Cruise are used for modeling and united simulation. The results show that the built model is accurate and reliable. The energy recovery rate can be improved effectively and the cruising range of pure electric vehicles can be extended based on proposed series regenerative braking control strategy.

In the article "Trusted computing in power distribution IoT: A fuzzy set theory based analysis"by Zhang et al., the authors relied on the comprehensive evaluation algorithm of fuzzy mathematics set theory and proposed a blockchain-based distributed decision-making and collaborative autonomy model for the power distribution IoT. The "manageable and controllable, precise protection, visible and credible, and smart defense" security protection model is established to carry out trusted computing and privacy protection of computer blockchain IoT nodes, and the information security defense level of the distribution network is improved comprehensively.

In the article "A fuzzy logic neural network algorithm for compressor crankshaft-rolling bearing system optimization" by Zhang et al., the authors used dynamic analysis software to conduct detailed dynamic analysis and optimal design under the rated power of the compressor. Using Hertz mathematical formula and the analysis method of the superstatic orientation problem, the relationship expression between the bearing force and deformation of the rolling bearing is solved, and the dynamic analysis model of the elastic crankshaft-rolling bearing system is constructed in the simulation software ADAMS. The weighted average amplitude of the center of the neck between the main bearings is used as the target, and the center line of the compressor cylinder is selected as the design variable.

In the article "Distribution optimization of electric vehicle load space based on interval type-ii fuzzy logic algorithm" by Zhang et al., the authors relied on the Interval Type-II fuzzy logic algorithm, the two optimization algorithm of interval type 2 fuzzy logic algorithm and genetic algorithm are compared from the space point of view, based on the research on the optimization problem of electric vehicle charging load space allocation, through the results of calculation examples in this paper. Practical results have verified the effectiveness and feasibility of the algorithm, and the interval two fuzzy logic algorithm has high practicability.

In the article "An improved AHP based fuzzy evaluation model for ship collision risk" by Luo et al., to evaluate the risk of ship collision, the authors analyzed the nature of the risk level of ship collision. Based on the fuzzy comprehensive evaluation model based on improved AHP, the concepts of space collision risk and time collision risk are proposed, and the models for space collision risk, time collision risk and collision risk level are established at the same time. The model developed and perfected the concept of ship collision risk, and are more logical, laid a solid foundation for subsequent comprehensive evaluation.

In the article "Dynamic simulation of wind field in three-dimensional display space considering particle swarm algorithm fuzzy logic" by Xu et al., in order to improve the accuracy of dynamic detection of wind field in the three-dimensional display space, the authors carried out system software on the actual scene and corresponding airborne radar observation information data, and the particle swarm algorithm fuzzy logic algorithm is introduced into the wind field dynamic simulation process in three-dimensional display space, to analyze the error of the filtering result in detail, to process the hurricane Lily Doppler radar measurement data with the optimal adaptive filtering according to the error data.

In the article "Applying fuzzy logic control to analyze real-time control for charging and discharging power of electric vehicles" by Liu et al., the authors proposed a real-time control method based on the clustering characteristics of the charging end time, according to the different charging requirements of the connected electric vehicles and fuzzy logic control is adopted to solve the problem of optimal charging and discharging power of the entire cluster and a single electric vehicle. A fuzzy logic control model considering the charging and discharging of electric vehicles is established orienting at minimize daily load fluctuations and control penalties in the upper layer. 
In the article "Dynamic control analysis of charging and discharging power for electric vehicles with adaptive optimal fuzzy control" by Meng et al., regarding the increasingly severe energy crisis and environmental issues, a dynamic control of the charging and discharging of electric vehicles into the grid that is universally adapted to optimal control is proposed by authors in this study. When the charging control system is based on the load function of the current distribution network, the electric vehicles connected to the charging station are charged and deployed, and the best fuzzy control is adopted to calculate the electric power to be transmitted to each charging hub, so as to improve the load characteristics of the regional grid to reach the grid The highest efficiency of use.

In the article "A RBF fuzzy logic neural network algorithm for construction resource scheduling" by Yu et al., the authors design a resource scheduling model for prefabricated building projects to optimize the scheduling of building resources. Analysis the constraint conditions between prefabricated construction projects. Then the radial basis function (RBF) fuzzy logic neural network algorithm was introduced. The results indicated that the method can help managers of prefabricated building projects carry out reasonable resource scheduling.

In the article "A multi-level fuzzy comprehensive assessment for supply chain risks" by Wang et al., the authors proposed a two-level fuzzy comprehensive assessment method for traffic enterprises based on the theory of fuzzy mathematics to access the potential supply chain risk. The data mainly derived from the expert assessment method. They use fuzzy cluster analysis to classify the indexes. Then more weighted indicators were obtained based on expert ratings, which were used as seeds for fuzzy evaluation at a higher level.

In the article "Autonomous driving of vehicles based on artificial intelligence" by Gao et al., the authors reviewed the application of artificial intelligence (AI) in the field of autonomous driving. Including the hardware and the software architecture of the autonomous driving system, the difference between the traditional vehicles and motors autonomous vehicles in the hardware part.

In the article "A rough set fuzzy logic algorithm for visual tracking of blockchain logistics transportation labels" by Liu et al., the authors put forward a fuzzy logic algorithm logistics based on the rough set visual image label tracking method to address the problems in the application process. The over- all visual features of the container are taken as the label feature, and the image segmentation transformation method is used to extract the features. The background is separated based on the key feature frames of the container images collected. The separated features are recorded, and the similarity of the features is used to complete the visual tracking.

In the article "Time-space evolution of the goods export network of the Belt and Road countries: the view of weighted complex network" by Liu et al., the authors used each country's bilateral exportation data to construct a weighted complex network of goods flows of the Belt and Road countries. Each country's centrality status in the network is measured by weighted centrality and PageRank index, and changes of each country's influence is measured by Hubbell influence index. Finally, the spatial clustering relationships and the correlation relationships among each block can be revealed by utilizing block model.

In the article "Research trends in computerized cognitive training contents with text network" by Lee et al., the authors collected literature published by PubMed, EMBASE, Cochrane Library, and Web of Science until December 2019 and analyze the trends of CCT and the transfer effect in each training area. In the literature that reported clinical effect (18/82), the cognitive domain mostly studied was memory (14/18), and the N-Back (3/14) method accounted for most of the training contents. Moreover, the contents that showed the highest degree, closeness, and betweenness centrality (BC) indices were the memory area, and video accounted for the highest among the intervention methods. In particular, the closeness centrality (CC) index of the memory and attention contents showed similar results.

In the article "Value co-creation mechanisms of multi-agent participation in crowdsourcing innovation", by Qingliang Meng et al., the authors analyzed the value co-creation realization mechanism of multiagent participation in the crowdsourcing innovation model from two aspects: the key influencing factors of value co-creation and the influence mechanism of the value co-creation process.

In the article "A deep metric learning approach for weakly supervised loan default prediction", by Kai Zhuang et al., the authors investigated how to address three challenges, namely insufficient default samples, hard decision boundaries and numerous heterogeneous features in loan prediction task and formulate this task as a weakly supervised loan default prediction problem, and propose a framework 
WEAKLOAN based on deep metric learning. The experimental results show that WEAKLOAN is superior to the compared baselines on both efficiency and effectiveness.

In the article "An urban metro network-based method to evaluate carbon emission and distribution cost of express delivery", by Junhua Guo et al., the authors proposed a multi-objective metro distribution model with the goal of minimizing metro operating costs and carbon emissions by using the $\mathrm{S} 1$ algorithm to solve this multi-objective GMED model. According to the characteristics of GMED, a two-layer coding method was constructed and substituted into NSGA-II for solution. The results of the case analysis show that GMED reduces carbon emissions and the mileage of vehicles compared to the vehicle express delivery (VED) method.

In the article "Optimization of security check efficiency in subway station based on anylogic", by Ming Wan et al., the authors used any logic software for simulation analysis to improve the efficiency of security check. The pedestrian density, the average number of queues and the number of people served are put forward as evaluation indexes. They found the bottleneck stage of the process is security check.

In the article "The impact of high-speed rail operations: a case study in Shanghai-Kunming line", by Cheng Zhang et al., the authors measured and quantitatively described the impact of high-speed railways on urban and regional spatial development. Through data analysis, the GDP growth rate of cities along the Shanghai-Kunming high-speed rail, the economic growth trend of the area passed by the ShanghaiKunming High-speed Railway is a U-shaped, which means opening of high-speed rail has increased the gap between core large cities and marginal small and medium-sized cities.

In the article "Analyzing influencing factors of crash injury severity incorporating FARS data", by Zhijian Zhang et al., the authors analyzed the influencing factors of driver's traffic accident casualties based on the relationship between the influencing factors of the accident and the injury severity, and established the Bayesian network and multinomial logit model. The Bayesian network reference analyses indicate that injury severity is affected by the crash factors, and there are various interactions between the various factors.

In the article "The impact of subway operation on urban traffic: A GRA-BNs based study", by Lixin Yan et al., the authors proposed a novel GRA-BNs method to analyze the impact of the subway operation on urban traffic, established a three-layer framework, and selected 18 traffic indicators from 22 traffic indicators by considering three aspects: traffic accessibility, green traffic, and traffic security.

In the article "Profit distribution of liner alliance based on Shapley value", by Guo et al., the authors established a new model by revising Shapley Value Method to distribute the profit of liner alliances from the perspectives of suppliers and customers and carry out verification through case analysis. The profit distribution method is helpful to the reasonable profit distribution of liner alliance. It ensures the continuity and stability of liner alliance and provides a scientific decision-making basis for the profit distribution of liner alliance.

In the article "Stochastic programming model and algorithm for flight schedule optimization" by Zhang et al., the authors proposed a two-stage stochastic programming model to solve the strategic flight schedule optimization problem for multiple airport and multiple operation days. Flight schedule optimization is made in the first stage of the model, and the tactical flight delay decision is made in the second stage with consideration of the impact of the uncertain operational airport capacity at the tactical stage. Then the authors designed a hybrid evolutionary algorithm to solve the model by two-stage decomposition.

In the article "A forest-fire model for technology risk transfer" by Wu et al., the authors constructed the system of systems (SoS) engineering process model of national major science and technology projects, then analyzed the hierarchical structure of national major science and technology projects, and divided the risks into different levels. Finally, the authors described the risk transfer mechanism using the forest-fire model and outlined a risk control strategy.

In the article "A fuzzy multi-objective programming optimization model for emergency resource dispatching under equitable distribution principle" by Tang et al., the authors proposed that the couple number of relief point-emergency point is the key to affect railway rescue cost and efficiency. Under the premise of the maximum satisfaction degree of quantity demanded at all emergency points, a multi-objective programming model is established by maximizing the satisfaction degree of dispatching time and the satisfaction degree of the couple number of relief point - emergency point. Combined with the ideal point method, a restrictive parameter interval method for optimal solution was designed, which 
can realize the quick seek of Pareto optimal solution.

In the article "A fuzzy analytic hierarchy process for risk evaluation of urban rail transit PPP projects" by Feng et al., the authors adopted the method of fuzzy analytic hierarchy process, token Shenzhen Metro Line 4 project as the research object and constructed the risk index system from nine dimensions to evaluate the risks of Public-Private Partnership project. Finally, the authors proposed the risk management system, risk sharing scheme and countermeasures in key risk areas.

In the article "A real-time train routing and platforming problem in complex railway stations" by Bai et al., the authors studied the real-time trains routing and platforming problem in railway stations that arises from the unreliable arrival times of freight trains, flexible shunting operations and dynamic station layout caused by equipment failure, and proposed an Integer Linear Program (ILP) that strives to minimize the number of conflicting trains.

In the article "Decision-making for location of manufacturing bases under uncertain demand situation" by Jianzhu Sun et al., this paper proposed a two-stage site selection model for multisite selection. Firstly, fuzzy number was used to represent the demand size of demand point. Secondly, the candidate manufacturing base with sufficient resources was selected based on the distance factor. Thirdly, a manufacturing base location model was established, which aimed at maximizing service efficiency under toke time, cost and demand as constraints. Finally, a stochastic numerical example is used to simulate the model.

In the article "A partner selection problem for complex product of manufacturing enterprises" by Congdong Li et al., aiming at the problem of partner selection for collaborative manufacturing of complex products in a collaborative supply chain environment, this paper proposed a multi-objective decision-making model that comprehensively considered the maximization of the matching degree of manufacturing capacity and the profits of supply chain. The method first used fuzzy theory to evaluate the manufacturing capabilities of candidate collaborative manufacturing partners. Secondly, Vector Space Model (VSM) is used to calculate the matching degree of manufacturing capacity and manufacturing demand. Then, the paper studied the profit of the supply chain under the "non-cooperative" mechanism and the "revenue sharing" mechanism. Furthermore, the decision-making model is established.
In the article "A simple and high accurate finite volume scheme for diffusion equations" by Zhucui Jing et al.., based on the "twin-fitting numerical methods" method, a simple and high accurate treatment for the vertex unknowns was developed and applied to the nine-point scheme for diffusion problem.

In the article "A Markov process model of sizing the capacity of the reserved storage yard in the intermodal container terminal" by Shujuan Guo et $a l$. , the authors proposed a Markov process model to explore the optimal capacity of the reserved storage yard in the intermodal container terminal. The flow balance equations were formulated to calculate the steady-state probability. The total cost is taken as the performance measure. Numerical analysis manifests that the railway preference rate and the strategy with and without transfer obviously affect the optimum capacity of the reserved storage yard. Some useful insights for the management of the intermodal container terminal are discussed.

In the article "Emergency evacuation with unbalanced utilization of exits at platform level: A Simulation study" by Chen et al., the authors took as an example that a subway station platform in Southwest China to establish a three-dimensional simulation model by using anylogic software. Through in-depth analysis, it is found that the reason for the unbalanced utilization of the platform floor exit is that the number of evacuation people at each exit does not match the evacuation capacity. According to the analysis results, the optimization model is put forward. Through comparative analysis, it is found that the optimization model can effectively balance the export utilization rate.

In the article "A capital input/output theory-based profit model for sharing bicycles" by Liu et al., the authors established the input and output value model of Sharing bicycles. In the big data era, it analyzes the acquired data using mathematical statistics, as well as using the Logit model to analyze the current Sharing bicycles model to evaluate profit conditions. The research results have shown that the Sharing bicycle has greatly enhanced people's green travel style since its launch, and it has great development potentials as well. The survey data shows that the Sharing bicycle adapts to the concept of green travel, low carbon, and environmental protection concept, which also has met the urgent demand for short-distance riding. This research provides effective ways and references for the future value-adding in the Sharing travel field. 
In the article "Feature selection by a distance measure method of subnormal and non-convex fuzzy sets" by Qu et al., the authors extended the general fuzzy set definitions to subnormal and non-convex fuzzy sets that are more precise. A distance measure method for subnormal and non-convex fuzzy sets is proposed for embedded feature selection. The weighted fuzzy membership functions prevent the combinatorial explosion of fuzzy rules in multiple fuzzy rule-based systems. The proposed method was validated by a comparison with two other methods. The method demonstrated higher accuracies in training and test, with scores of $97.95 \%$ and $93.98 \%$, respectively, compared to the other two methods.

In the article "Parking lots allocation model considering conversion capacity between car travelling and car parking" by Zhouhu Xie et al., targeted at the serious waste of parking resources, traffic congestion caused by too intensive parking demand in time and space, a parking allocation model considering the ability of dynamic and static traffic conversion is established with the goal of minimizing the total travel time of the travelers. Based on the dynamic traffic distribution model, the authors combine the dynamic and static traffic by considering the parking lot connection section as a conversion link to be added into the traffic network and propose a solution method based on particle swarm optimization.

In conclusion, the Guest Editors would like to thank all the authors who submitted their research articles to our special issue and would also like to acknowledge the guidance from the journal Editorin-Chief and staff members.

\section{Important dates}

Submission Deadline: 28 Feb, 2021

Acceptance/Rejection Notifications: 30 April, 2021

Final papers Due: 30 June, 2021 